\title{
Aggregate formation and radiolytic degradation of amphiphilic DNA block copolymer possessing disulfide bond.
}

\section{AUTHOR(S):}

Tanabe, Kazuhito; Asada, Takumi; Nishimoto, SeiIchi

\section{CITATION:}

Tanabe, Kazuhito ... [et al]. Aggregate formation and radiolytic degradation of amphiphilic DNA block copolymer possessing disulfide bond.. Bioorganic \& medicinal chemistry letters 2012, 22(23): 7045-7047

\section{ISSUE DATE:}

2012-12-01

URL:

http://hdl.handle.net/2433/162899

\section{RIGHT:}

(C) 2012 Elsevier Ltd.; This is not the published version. Please cite only the published version.; この論文は出版社版でありません。引用の際に は出版社版をご確認ご利用ください。 


\section{Aggregate formation and radiolytic degradation of amphiphilic DNA}

\section{block copolymer possessing disulfide bond}

Kazuhito Tanabe,* Takumi Asada, and Sei-ichi Nishimoto

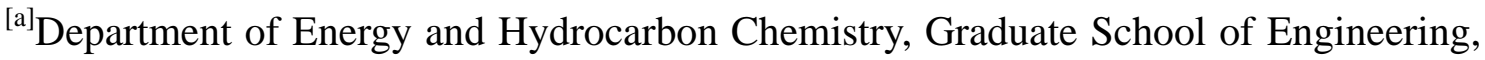

Kyoto University, Katsura Campus, Nishikyo-ku, Kyoto 615-8510, Japan

*corresponding author

Phone: +81-75-383-2505 FAX: +81-75-383-2504

e-mail: tanabeka@scl.kyoto-u.ac.jp 


\section{Graphical Abstract}

3'-Oligodeoxynucleotides-5' $-\mathrm{O}_{\text {Aggregate formation }}$
DNA block copolymer (DBC) \&

Degradation by $\mathrm{X}$-irradiation 


\section{Abstract}

We have designed a novel aggregate of DNA block copolymer (DBC) that is sensitive to hypoxic X-irradiation. The DBC consists of tetrahydropyrane-protected 2-hydroxyethyl methacrylate as a hydrophobic unit and oligodeoxynucleotides as a hydrophilic unit, which are linked to a radiation-sensitive disulfide bond. The DBC self-assembled efficiently to form aggregates that encapsulated small molecules such as nile red and pyrene. Hypoxic X-irradiation could then induce reductive degradation of the DBC aggregates via an exchange reaction of the disulfide bond to release guest molecules. 
There are increasing demands for effective molecular carrier systems, which can deliver drugs to target tissues in a selective manner to potentiate the effect of the drugs and eliminate any negative side effects. Of particular interest are nanoscale aggregates of amphiphiles with stimuli-responsive elements, because they can encapsulate drugs and the release them in the presence of prescribed stimuli. ${ }^{1,2}$ Heretofore, drug carrier systems, which are activated by photoirradiation, ${ }^{3-5}$ change of temperature ${ }^{6-9}$ or $\mathrm{pH}^{2,10,11}$ redox reaction ${ }^{12-14}$ and so forth, have been prepared.

High-energy ionizing radiation is an attractive stimulus for controlling a functions of biomaterials. $^{15-18}$ Ionizing radiation can control chemical reactions spatially and temporally without any additives. In particular, X-ray has potential of its high live-body permeability and thus has been extensively used for medical treatment and diagnosis. Recently, we demonstrated a degradation of drug carriers and release of drugs by means of hypoxic X-irradiation. ${ }^{19}$ We prepared DNA amphiphiles (DAMs) consisting of oligodeoxynucleotides (ODNs) as the hydrophilic unit and an alkyl chain as the hydrophobic unit, which were linked by a radiation-sensitive disulfide bond. ${ }^{20,21}$ DAMs assembled in aqueous solution to form nanosized aggregates that encapsulated 
hydrophobic drugs. X-irradiation induced an exchange reaction of the disulfide bond and thereby triggered a dissociation of the DAM aggregate and release of enclosed drugs. Although DAM aggregates were promising as radiation-activated drug carriers, DAMs showed large critical aggregation concentration (CAC) values, indicating that large amounts of DAMs were required to form drug carriers. In the light of minimization of cytotoxicity, drug carriers consisting of small amounts of amphiphiles are imperative.

The context of previous research prompted us to construct another type of amphiphile to improve its properties. We have now designed a DNA block copolymer (DBC), which consists of tetrahydropyrene-protected 2-hydroxyethyl methacrylate as the hydrophobic unit $^{1}$ and ODNs as the hydrophilic unit, which are linked by the disulfide bond (Figure 1). The DBC formed its aggregates in aqueous solution with a smaller CAC value than that of DAMs, and encapsulated a hydrophobic dye as a virtual drug. The DBC aggregates were readily degraded by hypoxic X-irradiation to release the encapsulated dyes.

An amphiphilic DBC possessing disulfide bond was prepared by coupling of a 
hydrophobic polymer (HP) and 30 mer ODNs possessing a thiol group at the 5'-end according to the previous report. ${ }^{1}$ The crude product was purified by HPLC and the formation of the DBC was determined by MALDI-TOF mass spectrometry. ${ }^{22}$

Aggregation of the DBC was evaluated using a fluorescence probe of nile red as a hydrophobic dye. ${ }^{23}$ Nile red shows no fluorescence emission in an aqueous medium because of its poor solubility, whereas it shows intense fluorescence, even in water, when it is solubilized into the hydrophobic interior of aggregates consisting of amphiphiles. ${ }^{1}$ Therefore, we can monitor the aggregation of the DBC by fluorescence emission. As shown in Figure 2A, intense fluorescence of nile red was observed in the presence of aggregate-forming DBC even in water, but was dramatically suppressed in the presence of either the hydrophilic 30 mer ODN unit or the HP alone. These results strongly indicate that the DBC formed aggregates in water and encapsulated the nile red. In a separate experiment, we conducted enzymatic digestion of the DNA unit of the aggregates and monitored the fluorescence change (Figure 2B). After treating the aggregates with nuclease P1, phosphodiesterase, and alkaline phosphatase, which convert ODNs into monomeric nucleosides, the fluorescence emission was dramatically 
suppressed because of the decomposition of the aggregates. Thus, the hydrophilic unit of ODNs in the DBC is indispensable for aggregate formation.

We next determined the CAC of DBC using nile red. The concentration of DBC was varied and the change in the relative emission intensity was plotted. As shown in Figure 3A, a sudden increase in fluorescence intensity was observed at a DBC concentration at $21 \mathrm{nM}$, which was assigned to the CAC. Given that the CAC value of the previous DAM was 2.3-8.8 $\mu \mathrm{M}$, the long and bulky hydrophobic unit in DBC is critical for the formation of stable aggregates. To verify further the formation of the DBC aggregates and measure the size of the assembly, we conducted transmission electron microscopy (TEM) image analysis and dynamic light scattering (DLS) experiments. Figure 3B shows a representative TEM image of the aggregates. The DBC formed aggregates with spherical and elliptical shapes with a diameter of $101 \pm 10 \mathrm{~nm}$. Alternatively, the diameters of aggregates in water measured by DLS were estimated to be $194 \pm 49 \mathrm{~nm}$ (Figure 3C). These results clearly indicate that the DBC formed aggregates of more than $100 \mathrm{~nm}$ diameter.

An attempt was also made to demonstrate the radiolytic decomposition of the DBC 
aggregates and the release of the functional molecules encapsulated in the aggregates. ${ }^{24}$

The hydrophobic unit and the hydrophilic unit in DBC were linked by radiation-sensitive disulfide bond. ${ }^{20,21}$ We therefore predicted that radiolytic reduction would induce a degradation of the aggregates because the exchange reaction at the disulfide bond would destroy the balance between the hydrophilic and hydrophobic units in the DBC. We performed radiolytic reduction of the DBC aggregates in argon-purged aqueous solution containing an excess amount of 2-methyl-2-propanol under hypoxic conditions. Reducing hydrated electrons and hydrogen atoms $\left(\mathrm{H}^{\circ}\right)$ are thus generated as the major active species under these radiolysis conditions, where oxidizing hydroxyl radicals $\left(\mathrm{OH}^{*}\right)$ may be effectively scavenged by the 2-methyl-2-propanol. ${ }^{25}$ To monitor the radiolysis, we employed a fluorescent pyrene molecule as the functional molecule encapsulated in the aggregates, because nile red is unstable to radiation. Figure 4 shows representative fluorescence spectra of pyrene in the aggregates. We observed strong fluorescence around $400 \mathrm{~nm}$ from the sample without irradiation, while the fluorescence intensity of pyrene decreased with increasing radiation dose. This result indicates that hypoxic X-irradiation degraded the DBC 
aggregates, resulting in a release of pyrene and a decrease in fluorescence emission. The amount of pyrene released from the DBC aggregates was estimated to be $99 \mathrm{nM}$ after 360 Gy of hypoxic X-irradiation. Thus, it is reasonable to conclude that DBC formed efficiently its aggregates, which were easily degraded by $\mathrm{X}$-irradiation to release enclosed molecules.

In conclusion, we prepared an amphiphilic DNA block copolymer possessing disulfide bond (DBC) and characterized its properties as a radiation-activated drug carrier. The DBC consisted of hydrophilic ODNs and hydrophobic polymer unit self-assembled to form aggregates in water and thereby to solubilize a hydrophobic fluorescent dyes by encapsulation. According to the CAC measurements, the DBC formed more stable aggregates than the previous amphiphile DAM with an alkyl chain as the hydrophobic unit. Hypoxic X-irradiation of DBC aggregates in aqueous solution activated the disulfide bond in the DBC, resulting in its decomposition and the release of the fluorescent dye. Thus, DBC aggregates represent a promising prototype compound for a novel radiation-controlled drug delivery system. Encapsulation of actual drugs and cytotoxicity evaluation of DBC aggregates are in progress. 
Acknowledgement: This work is partly supported by the Research Grant Program from Asahi Glass foundation and by the Strategic Promotion Program for Basic Nuclear Research from the Ministry of Education, Culture, Sports, Science and Technology (MEXT), Japan. 


\section{References}

(1) Klaikherd, A.; Nagamani, C.; Thayumanavan, S. J. Am. Chem. Soc. 2009, 131, 4830.

(2) Chiu, H. -C.; Lin, Y. -W.; Huang, Y. -F.; Chuang, C. -K.; Chern, C. -S. Angew. Chem. Int. Ed. 2008, 47, 1875.

(3) Nishiyama, N.; Iriyama, A.; Jang, W. -D.; Miyata, K.; Itaka, K.; Inoue, Y.; Takahashi, H.; Yanagi, Y.; Tamaki, Y.; Koyama, H.; Kataoka, K. Nat. Mater. 2005, 4, 934.

(4) Goodwin, A. P.; Mynar, J. L.; Ma, Y.; Fleming, G. R.; Fréchet, J. M. J. J. Am. Chem. Soc. 2005, 127, 9952.

(5) Jiang, J.; Tong, X.; Zhao, Y. J. Am. Chem. Soc. 2005, 127, 8290.

(6) Sundararaman, A.; Stephan, T.; Grubbs, R. B. J. Am. Chem. Soc. 2008, 130, 12264.

(7) Oupický, D.; You, Y. -Z. Biomacromolecules 2007, 8, 98.

(8) Morishima, Y. Angew. Chem. Int. Ed. 2007, 46, 1370.

(9) Aathimanikandan, S. V.; Savariar, E. N.; Thayumanavan, S. J. Am. Chem. Soc. 
2005, 127, 14922.

(10) Auguste, D. T.; Furman, K.; Wong, A.; Fuller, J.; Armes, S. P.; Deming, T. J.; Langer, R. J. Controlled Release 2008, 130, 266.

(11) Du, J.; Tang, Y.; Lewis, A. L.; Armes, S. P. J. Am. Chem. Soc. 2005, 127, 17982.

(12) Takae, S.; Miyata, K.; Oba, M.; Ishii, T.; Nishiyama, N.; Itaka, K.; Yamasaki, Y.; Koyama, H.; Kataoka, K. J. Am. Chem. Soc. 2008, 130, 6001.

(13) Stevenson, M.; Ramos-Perez, V.; Singh, S.; Soliman, M.; Preece, J. A.; Briggs, S. S.; Read, M. L.; Seymour, L. W. J. Controlled Release 2008, 130, 46.

(14) Napoli, A.; Valentini, M.; Tirelli, N.; Muller, M.; Hubbell, J. A. Nat. Mater. 2004, 3, 183.

(15) Spinks, J. W. T.; Wood, R. J. Introduction to Radiation Chemistry 3rd Ed.; Wiley-Interscience: New York, 1990.

(16) Tanabe, K.; Zhang, Z.; Ito, T.; Hatta, H.; Nishimoto, S. Org. Biomol. Chem. 2007, 5, 3745 .

(17) Ito, T.; Tanabe, K.; Yamada, H.; Hatta, H.; Nishimoto, S. Molecules 2008, 13, 2370. 
(18) Ma, N.; Xu, H.; An, L.; Li, J.; Sun, Z.; Zhang, X. Langmuir 2011, 27, 5874.

(19) Tanabe, K.; Asada, T.; Ito, T.; Nishimoto, S. Bioconjugate Chem. Articles ASAP, DOI: $10.1021 / \mathrm{bc} 3002985$.

(20) Tanabe, K.; Kuraseko, E.; Yamamoto, Y.; Nishimoto, S. J. Am. Chem. Soc. 2008, 130, 6302.

(21) Tanabe, K.; Matsumoto, E.; Ito, T.; Nishimoto, S. Org. Biomol. Chem. 2010, 8, 483.

(22) The formation of DBC was identified by measurement of MALDI-TOF mass spectrometry. [M-H] found 13157.0. (Molecular weight of thiol modified ODNs was 9260.0).

(23) General procedure for the formation of aggregates consisted of DBC and Fluorescence measurements: To form aggregate, DBC $(5.5 \mu \mathrm{M})$ dissolved in water was added to nile red $(10 \mu \mathrm{M})$ in acetonitrile. Then, the solvent was removed in vacuo. The resulting mixture was dissolved in water to form aggregate, in which nile red was encapsulated. Fluorescence spectra were taken with excitation at $560 \mathrm{~nm}$. 
(24) General procedure for radiolytic reduction of DBC aggregate: To establish hypoxia, the aggregates consisted of DBC (680 nM) encapsulating pyrene dye $(10 \mu \mathrm{M})$ in water containing 10\% 2-methyl-2-propanol were purged with argon for $10 \mathrm{~min}$ and then irradiated in a sealed glass ampoule at ambient temperature with an X-ray source (6.0 Gy $\left.\mathrm{min}^{-1}\right)$. Before or after the irradiation, the solution was subjected to fluorescence measurements. The fluorescence spectra were measured at $342 \mathrm{~nm}$ excitation.

(25) Buxton, G. V.; Greenstock, C. L.; Helman, W. P.; Ross, A. B. J. Phys. Chem. Ref. Data 1988, 17, 513. 

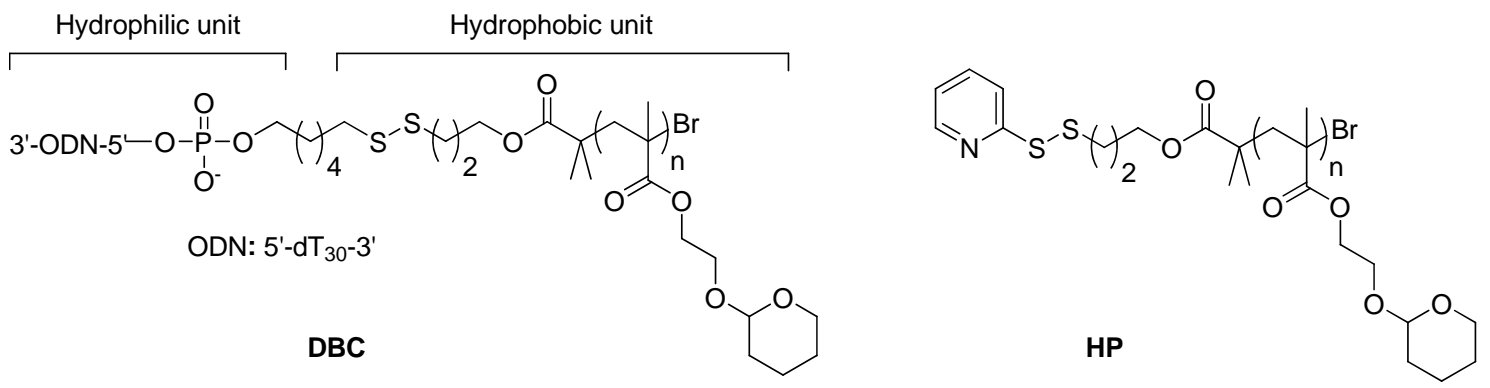

Figure 1. Sequences and structure of amphiphilic DNA block copolymer (DBC) and hydrophobic polymer (HP). 
(A)

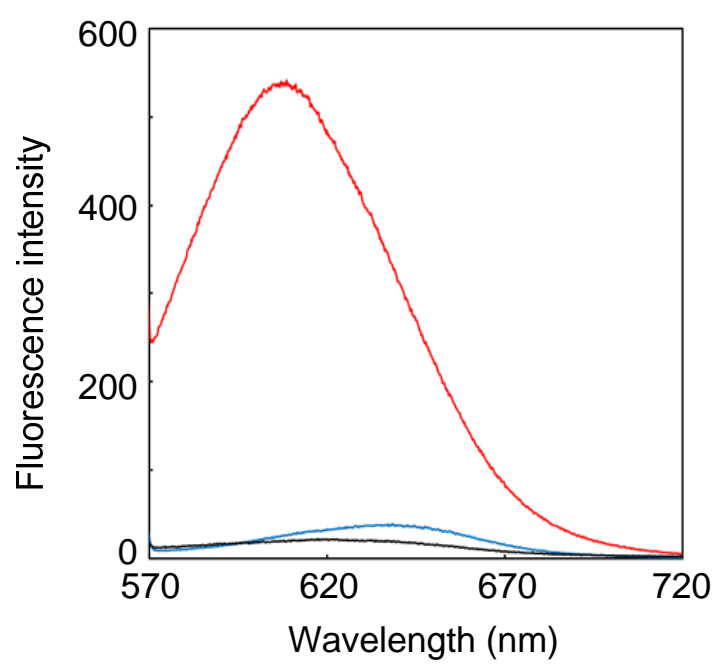

(B)

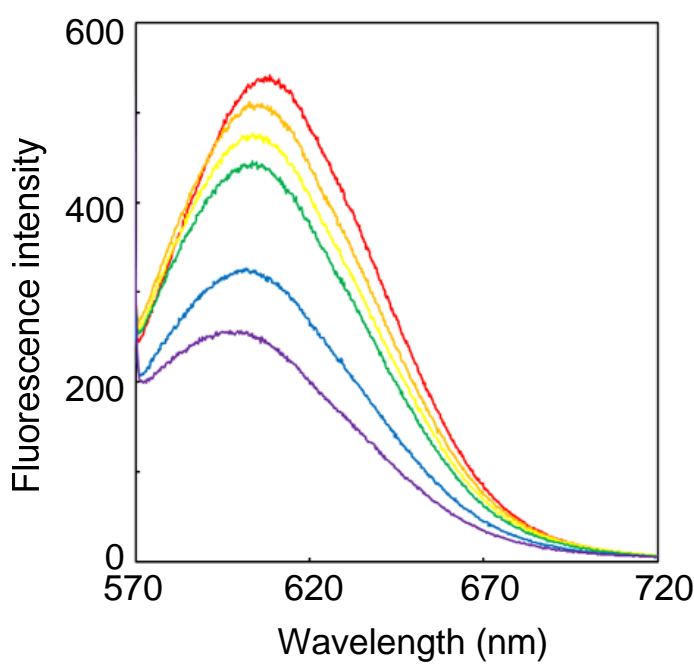

Figure 2. (A) Fluorescence spectra of nile red $(10 \mu \mathrm{M})$ in water in the presence of DBC (5.5 $\mu \mathrm{M}$, red), 30 mer ODNs ( $\mathrm{dT}_{30}, 130 \mu \mathrm{M}$, blue), or P1 (10 ng / mL, black). (B) Fluorescence spectra of nile red $(10 \mu \mathrm{M})$ in the presence of DBC (5.5 $\mu \mathrm{M})$ after treatment with nuclease P1, phosphodiesterase and alkaline phosphatase for 0 (red), 3 (orange), 6 (yellow), 12 (green), 24 (blue) and $48 \mathrm{~h}$ (purple) at $37^{\circ} \mathrm{C}$. 
(A)

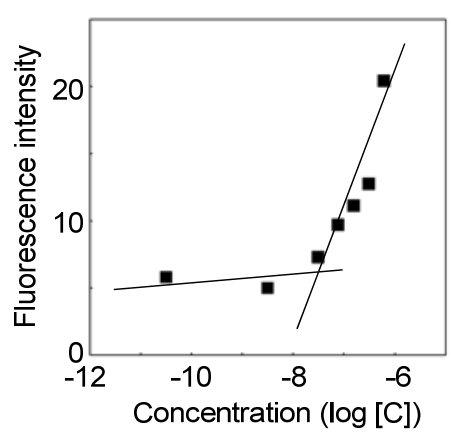

(B)

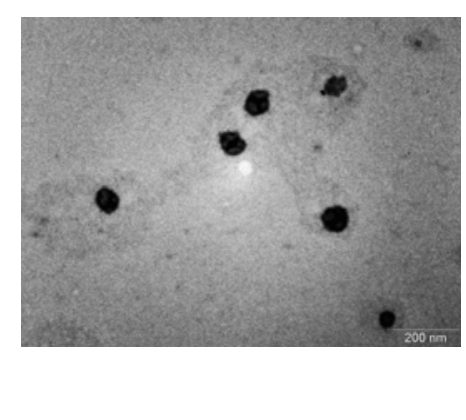

(C)

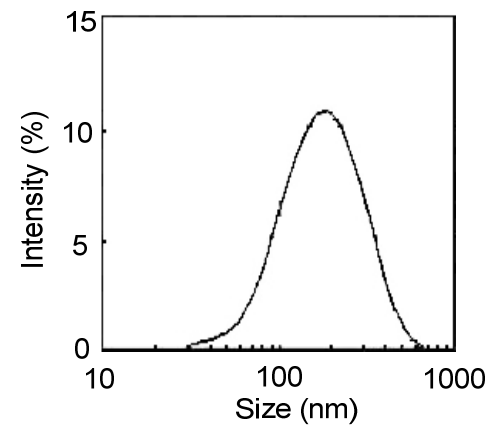

Figure 3. (A) Plot of fluorescence intensity of nile red $(10 \mu \mathrm{M})$ vs concentration of DBC. (B) TEM images of aggregate consisted of DBC $(1.6 \mu \mathrm{M})$. (B) Size of the aggregate consisted of DBC $(1.6 \mu \mathrm{M})$ determined by DLS experiment. 


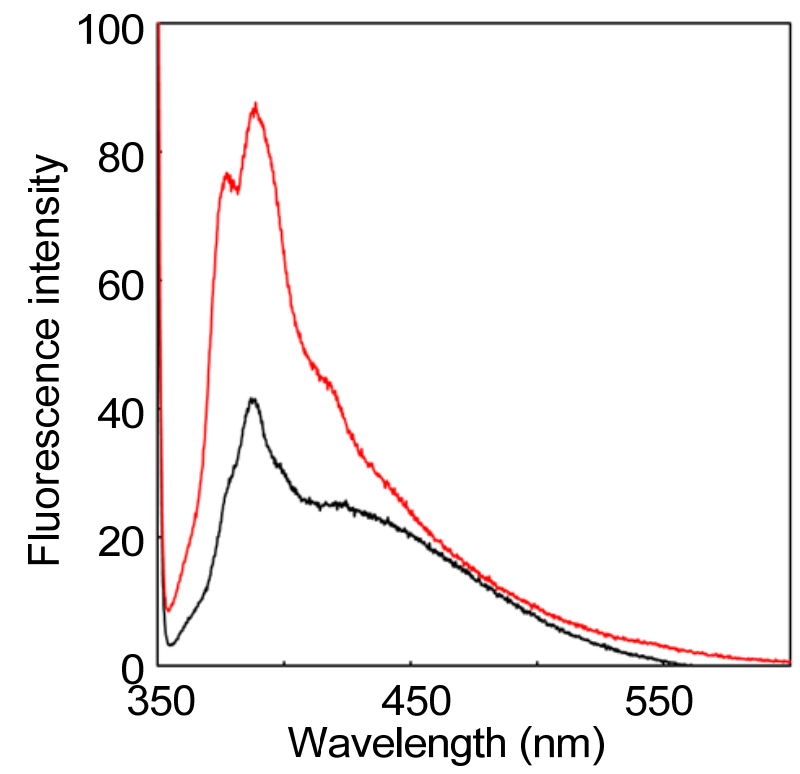

Figure 4. Fluorescence spectra of pyrene $(10 \mu \mathrm{M})$ in water containing $10 \%$ 2-methyl-2-propanol in the presence of DBC (A, $680 \mathrm{nM})$. Spectra were measured after hypoxic X-irradiation (0 Gy: red, 360 Gy: black). 